Gefässchirurgie 2022 $27: 380-387$

https://doi.org/10.1007/s00772-022-00864-7

Angenommen: 7. Januar 2022

Online publiziert: 8. Februar 2022

(c) Der/die Autor(en) 2022

\title{
Erste Erfahrungen mit der Bio- Zellulose Nanogen Aktiv in der Behandlung komplexer Wunden
}

Rio Marnoto · Tobias Dominik Warm • Kerstin Storl · Alexander Hyhlik-Dürr · Yvonne Nicole Goßlau

Gefäßchirurgie und endovaskuläre Chirurgie, Medizinische Fakultät, Universität Augsburg, Augsburg, Deutschland

\section{Zusammenfassung}

Hintergrund: Komplexe Wundsituationen stellen Patient und Therapeut häufig vor eine große Herausforderung. Neben der Behandlung der ursächlichen Erkrankung ist die Lokaltherapie bei der Wundbehandlung essenziell. Neben den klassischen Wundauflagen stehen mittlerweile mit Nanomaterialien neue Optionen zur Verfügung. Fragestellung: Die vorliegende Studie untersucht den Einfluss von Nanogen Aktiv (Vertrieb Lamed, Oberhaching, Deutschland) auf die Wundheilung und erfasst die Patientenzufriedenheit am Ende der Behandlung.

Material und Methoden: In der prospektiven Single-Center-Studie wurden im Zeitraum von 02/18 bis 04/20 insgesamt 20 Patienten mit chronischen Wunden mit Nanogen Aktiv behandelt. Hierzu wurden demografische Daten, das Keimspektrum in den Wunden und klinische Verläufe erfasst und ausgewertet.

Ergebnisse: In sieben Fällen (7/20) konnte eine Reduktion der Wundfläche in 4-40 Wochen erreicht werden. Bei den übrigen 13 Patienten konnte aus verschiedenen Gründen kein Therapieerfolg erzielt werden.

Diskussion: Nanogen Aktiv kann einen positiven Effekt auf die Heilung komplexer Wunden haben. Größe und Tiefe der Wunde beeinflussen die Therapiedauer. Die zugrunde liegende Erkrankung muss zwingend mitbehandelt werden. Die Indikation zur Behandlung mit Nanogen Aktiv sollte aufgrund des hohen zeitlichen Aufwandes individuell gestellt werden.

\section{Schlüsselwörter}

Wundbehandlung $\cdot$ Extrazelluläre Matrix · Chronische Wunden · pAVK · Ulcus

\section{Hintergrund und Fragestellung}

Komplexe Wunden zählen zu den häufigsten chronischen Erkrankungen in Deutschland. Der Begriff der komplexen Wunde wird in der vorgestellten Untersuchung für chronische Wunden mit protrahiertem Heilungsverlauf und/oder Therapieresistenz verwendet.

Zu den häufigsten Ursachen zählen vaskuläre Grunderkrankungen, wie die chronisch venöse Insuffizienz (CVI; 57-80\% der Fälle) oder die periphere arterielle Verschlusskrankheit (pAVK; 4-30\% der Fälle). Bei $10 \%$ der Fälle handelt es sich um gemischt arterio-venöse Pathologien [1].

Neben erheblicher Belastung des Gesundheitssystems durch die Behandlungs- kosten und Bindung von Ressourcen steht besonders die Einschränkung der Lebensqualität der betroffenen Patienten im Fokus. Trotz Optimierung der Lokaltherapie und Behandlung der zugrunde liegenden Erkrankung ist der Heilungsprozess oft langwierig [4].

Weitere Ursachen stellen unter anderem Mangelernährungszustände, Tumoren, Vaskulitiden und chronische Entzündungen dar, die in ihrem Krankheitsverlauf einen negativen Einfluss auf die Wundheilung nehmen können [10]. Neben dem langwierigen Therapieverlauf chronischer Wunden und der damit verbundenen deutlichen Einschränkung der Lebensqualität der Patienten können sie auch schwerwiegende Komplikationen, wie 
Tab. 1 Darstellung der demografischen Daten, der zugrunde liegenden Erkrankung und deren Behandlung Patientencharakteristika

\begin{tabular}{|c|c|c|c|c|c|c|}
\hline ID & Alter & Geschlecht & Wundlokalisation & Grunderkrankung & Vaskuläre Therapie & CVRF \\
\hline 1 & 66 & w & Fuß & pAVK & Endovaskuläre Versorgung & aHT, DM, KHK, Nikotin, NI \\
\hline 2 & 70 & $\mathrm{~m}$ & Fuß & pAVK & Endovaskuläre Versorgung & aHT, DM, Nikotin \\
\hline 3 & 83 & $\mathrm{~m}$ & Unterschenkel & pAVK & Endovaskuläre Versorgung & aHT, DM, NI \\
\hline 4 & 85 & $\mathrm{~m}$ & Fuß & Posttraumatisch bedingt & Keine & aHT \\
\hline 5 & 76 & $\mathrm{~m}$ & Unterschenkel & pAVK & Femorocrurale Bypassanlage & aHT, NI \\
\hline 6 & 75 & w & Unterschenkel & Unklare Genese & Keine Therapie & aHT, Nikotin \\
\hline 7 & 66 & $\mathrm{~m}$ & Unterschenkel & pAVK & Femorocrurale Bypassanlage & aHT, KHK \\
\hline 8 & 69 & $\mathrm{~m}$ & Oberschenkel & pAVK & Femoropopliteale Bypassanlage & aHT, Nikotin \\
\hline 9 & 69 & w & Unterschenkel & pAVK & Leisten-TEA & aHT, DM \\
\hline 10 & 69 & $\mathrm{~m}$ & Fuß & pAVK & Femoropopliteale Bypassanlage & aHT, Nikotin \\
\hline 11 & 67 & w & Unterschenkel & pAVK & Femorocrurale Bypassanlage & $\mathrm{aHT}$ \\
\hline 12 & 63 & $\mathrm{~m}$ & Fuß & Neuropathisches DFS & Keine & DM \\
\hline 13 & 84 & $w$ & Unterschenkel & pAVK & Endovaskuläre Versorgung & aHT, KHK \\
\hline 14 & 66 & $\mathrm{~m}$ & Unterschenkel & pAVK & Femoropopliteale Bypassanlage & aHT, KHK, Nikotin, NI \\
\hline 15 & 86 & $\mathrm{~m}$ & Oberschenkel & pAVK & Femoropopliteale Bypassanlage & aHT, DM, Nikotin, NI \\
\hline 16 & 82 & w & Unterschenkel & Unklare Genese & Keine & $\mathrm{NI}$ \\
\hline 17 & 63 & $\mathrm{~m}$ & Fuß & Unklare Genese & Keine & $\mathrm{aHT}$ \\
\hline 18 & 50 & $w$ & Leiste & pAVK & Femoropopliteale Bypassanlage & aHT, DM \\
\hline 19 & 50 & $\mathrm{~m}$ & Fuß & Neuropathisches DFS & Keine & aHT, DM \\
\hline 20 & 78 & $\mathrm{~m}$ & Oberschenkel & pAVK & Endovaskuläre Versorgung & aHT, DM \\
\hline
\end{tabular}

septische Verläufe, zur Folge haben. Ein weiteres Patientenkollektiv, das besonders stark durch chronische Wunden gefährdet ist, stellen Diabetiker dar. Auch wenn in Deutschland zwischen 2005 und 2014 der Anteil der Majoramputationen bei Patienten mit diagnostiziertem Diabetes mellitus an der Gesamtzahl der Majoramputationen von 70,2 auf $63,7 \%$ sank [6], stellt das diabetische Fußsyndrom mit 2-10\% Prävalenz eine häufige Ursache für den Extremitätenverlust dar [1].

Die Lokaltherapie ist neben der Behandlung der zugrunde liegenden Erkrankung essenziell. Gerade im Bereich der lokalen Wundtherapie steht dem Therapeuten eine große Auswahl von Wundauflagen verschiedener Hersteller zur Verfügung. Die unterschiedlichen Lokaltherapeutika sollen jeweils phasenadaptiert angewendet werden. Eine besondere Rolle spielt hierbei das Prinzip der modernen "feuchten“ Wundbehandlung $[2,11]$. Hierunter versteht man die Versorgung der Wunde mit Auflagen, die ein feuchtes Wundmilieu entstehen lassen. Als Pionier der modernen Wundtherapie konnte Winter in den Sechzigerjahren belegen, dass artifizielle Wun- den beiSchweinen durch Applikation eines okklusiven Folienverbandes beschleunigt abheilen [12]. Im Unterschied zu trockenen Wundauflagen verbleiben diese „modernen Verbände" länger auf der Wunde und leisten dadurch auch einen Beitrag zur Lebensqualität der Patienten aufgrund der geringeren Frequenz der Verbandswechsel.

Nanogen Aktiv und Aktigel (Vertrieb Lamed, Oberhaching, Deutschland) stellen eine solche moderne Wundauflage dar. Die sich ergänzenden Produkte sollten zusammen angewendet werden und bestehen zu $100 \%$ aus natürlichem Nanofasermaterial, welches rein pflanzlich hergestellt wird und ausschließlich im brasilianischen Regenwald vorkommt. Die Nanomembran wird mittels eines 20-tägigen Produktionsprozesses in einem Bioreaktor hergestellt. In der Wunde verteilt diese Membran Nanofasern $(<2 \mathrm{~nm})$, die eine effektive extrazelluläre Matrix bilden. Nanogen Aktigel ist eine Substanz, die aus demselben Pflanzenextrakt und denselben Stammzellen wie Nanogen Aktiv hergestellt wird. Die Gelform fördert eine schnelle Absorption durch das Wundbett. Die Membran und das Gel von Nanogen Aktiv sollen eine fehlende oder fehlerhafte extrazelluläre Matrix ersetzen oder reparieren. Zusätzlich unterstützt es die interzelluläre Signalübertragung, wodurch die für die Wundheilung wichtigen Zellen wie Fibroblasten und Keratinozyten angeregt werden, ihre Reparaturarbeiten fortzusetzen [3].

Ziel der vorliegenden Studie ist die prospektive Analyse der Anwendung von Nanogen Aktiv und Aktigel bei der Behandlung komplexer Wunden. Primärer Endpunkt ist dabei die Reduktion der Wundoberfläche durch Epithelisierung. Weiterhin wurde die Erhebung von subjektiver Patientenzufriedenheit bei der Therapie dokumentiert sowie die Änderung des Keimspektrums erfasst.

Zur Durchführung der Studie wurden die gesetzlichen Bestimmungen zum Datenschutz eingehalten. Eine Auswertung von Patientendaten erfolgte nur bei vorliegender "Einwilligungserklärung zum Datenschutz". 


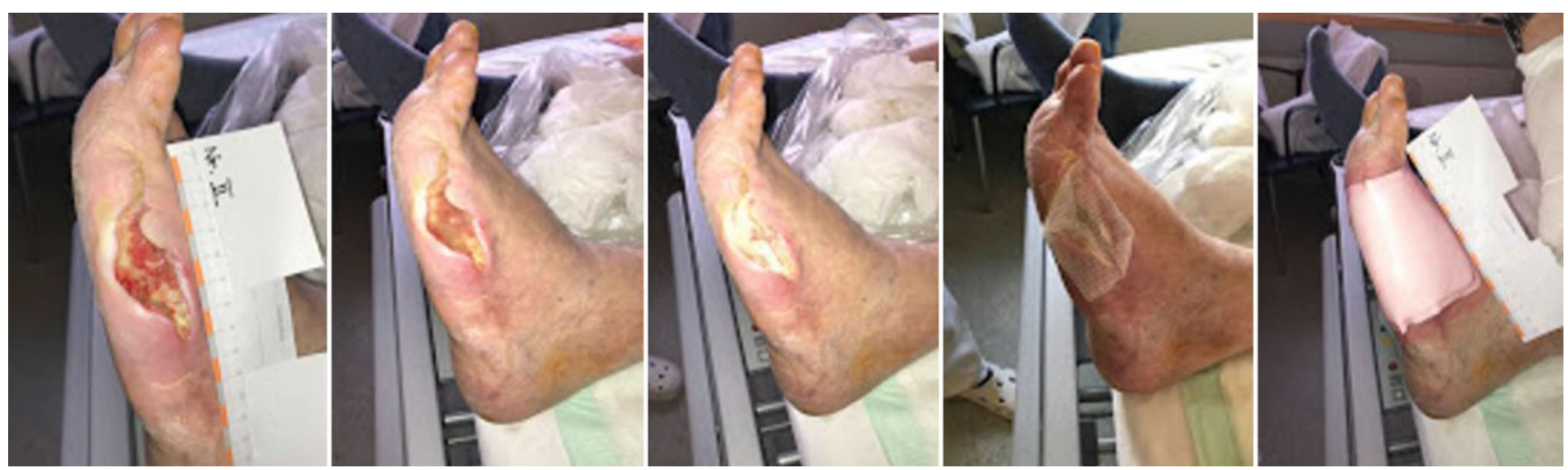

Abb. 1 ム Anlage der Nanogen-Aktiv-Membran auf die Wunde. Bei Therapiebeginn und jedem Verbandswechsel erfolgt ein Débridement

\section{Studiendesign und Untersuchungsmethoden}

Am Universitätsklinikum Augsburg wurden zwischen Februar 2018 und April 2020 insgesamt 20 Patienten ( $w=7, m=13)$ mit Nanogen Aktiv behandelt. Die Patienten waren im Mittel 71,1 Jahre (50-86) alt. Als Einschlusskriterium galt das Vorliegen einer komplexen Wundsituation; diese wurde definiert als chronische Wunde unterschiedlicher Genese, wie beispielsweise bei venöser und/oder arterieller Grunderkrankung, bei diabetischem Fußsyndrom sowie bei Wundheilungsstörung nach Amputation oder Infektspaltung. Die Wundbehandlung erfolgte bis zum Einschluss in die vorliegende Studie mit etablierten hydroaktiven Wundverbänden, welche nicht zu einer Besserung der Wundsituation führten. Der genaue Zeitrahmen der Vorbehandlung konnte retrospektiv nicht erfasst werden.

Die Therapie der Grunderkrankung musste vor der Rekrutierung in die Studie erfolgreich abgeschlossen werden. Um dies zu bestätigen, wurde bei allen Patienten bei Studienbeginn die Durchblutungssituation durch Bestimmung des $\mathrm{ABI}$ (Ankle-Brachial-Index) oder durch eine $\mathrm{tcpO}_{2}$-Messung (transkutane Sauerstoffpartialdruckmessung) evaluiert. Ein venöses FKDS (farbkodierte Duplexsonographie) erfolgte bei klinischen Anzeichen einer chronisch venösen Insuffizienz.

Alle Patienten mit pAVK im Stadium IV $(n=14)$ wurden arteriell revaskularisiert. Es erfolgten fünf perkutane endovaskuläre Interventionen, eine operative Rekonstruktion der Femoralisgabel durch Throm- bendarteriektomie und in acht Fällen Bypassanlagen (femoropopliteal bzw. femorocrural). Bei zwei Patienten lag ein neuropathisches diabetisches Fußsyndrom ohne vaskuläre Beteiligung vor, weshalb sich die Therapie auf eine lokale Wundversorgung beschränkte. Ein Patient wies eine chronische Wunde nach lokalem Trauma auf. Bei drei Wunden konnte keine arterielle oder venöse Genese nachgewiesen werden; auch nach histologischer Untersuchung ergaben sich keine spezifischen Ursachen. Bei den Wunden mit nachvollziehbarer Genese erfolgte keine Biopsie (- Tab. 1).

Initial erfolgte bei allen Wunden ein chirurgisches Débridement und eine $A b$ strichentnahme zur mikrobiologischen Testung unter aseptischen Bedingungen. Eine Fotodokumentation zur Beurteilung des Heilungsverlaufes mit Messung der Wundausdehnung wurde ebenfalls durchgeführt.

Anschließend wurde zur Keimreduktion Silgen Ag Silberspray (Genadyne Pharma) auf die Wunde gesprüht und nach einer Einwirkzeit von circa zwei Minuten das Nanogen Aktigel auf die Wunde aufgetragen. Die Nanogen-AktivMembran wird entsprechend der Wundgröße zurechtgeschnitten und auf das Gel in der Wunde gelegt. Darüber wird ein Silikon-Wunddistanzgitter angebracht (Mepitel ${ }^{\circledR}$, Mönlycke Health Care, Gotenberg, Schweden) sowie ein SilikonSchaumverband (Mepilex ${ }^{\circledR}$ Border, Mönlycke Health Care, Gotenberg, Schweden) zur Abdeckung aufgelegt (• Abb. 1).

Die weiteren Verbandswechsel erfolgten ambulant ein- bis zweimal pro Wo- che. Die Wunden wurden dabei jeweils gesäubert bzw. débridiert und fotodokumentiert. Abschließend erfolgte die Verbandsauflage nach beschriebenem Schema.

Alle zwei Wochen wurde eine mikrobiologische Testung mittels Abstrichentnahme durchgeführt, um die Auswirkungen der Therapie auf das Keimspektrum der Wunde zu dokumentieren. Am Ende der Therapie wurde die Zufriedenheit der Patienten mit der Behandlung ermittelt. Hierzu wurde eine analoge zehnstufige Ratingskala verwendet, die von 1 (ungenügend) bis 10 (sehr gut) reichte. Die Datenerfassung erfolgte über einen eigens erstellten Fragebogen. Die Dokumentation und statistische Auswertung erfolgte mit Microsoft Excel (Version 2010; Microsoft Corporation, Redmond, WA, USA).

\section{Ergebnisse}

Das beschriebene Schema aus Débridement und regelmäßigen Verbandswechseln mit Nanogen Aktiv wurde bei $20 \mathrm{~Pa}$ tienten angewendet.

Die Wunden waren allesamt an der unteren Extremität lokalisiert; sieben am Fuß, neun am Unterschenkel, drei am Oberschenkel und eine Wunde in der Leiste.

Bei acht Wunden konnten eine Reduktion der Wundoberfläche festgestellt werden, davon erreichten sieben die Epithelisierungsphase (Beispiele - Abb. 2).

Bei der achten Wunde wurde die Behandlung auf Wunsch des Patienten nicht weiter fortgesetzt, obwohl sich die Wunde zum Zeitpunkt des Therapieabbruchs bereits in der proliferativen Granulations- 

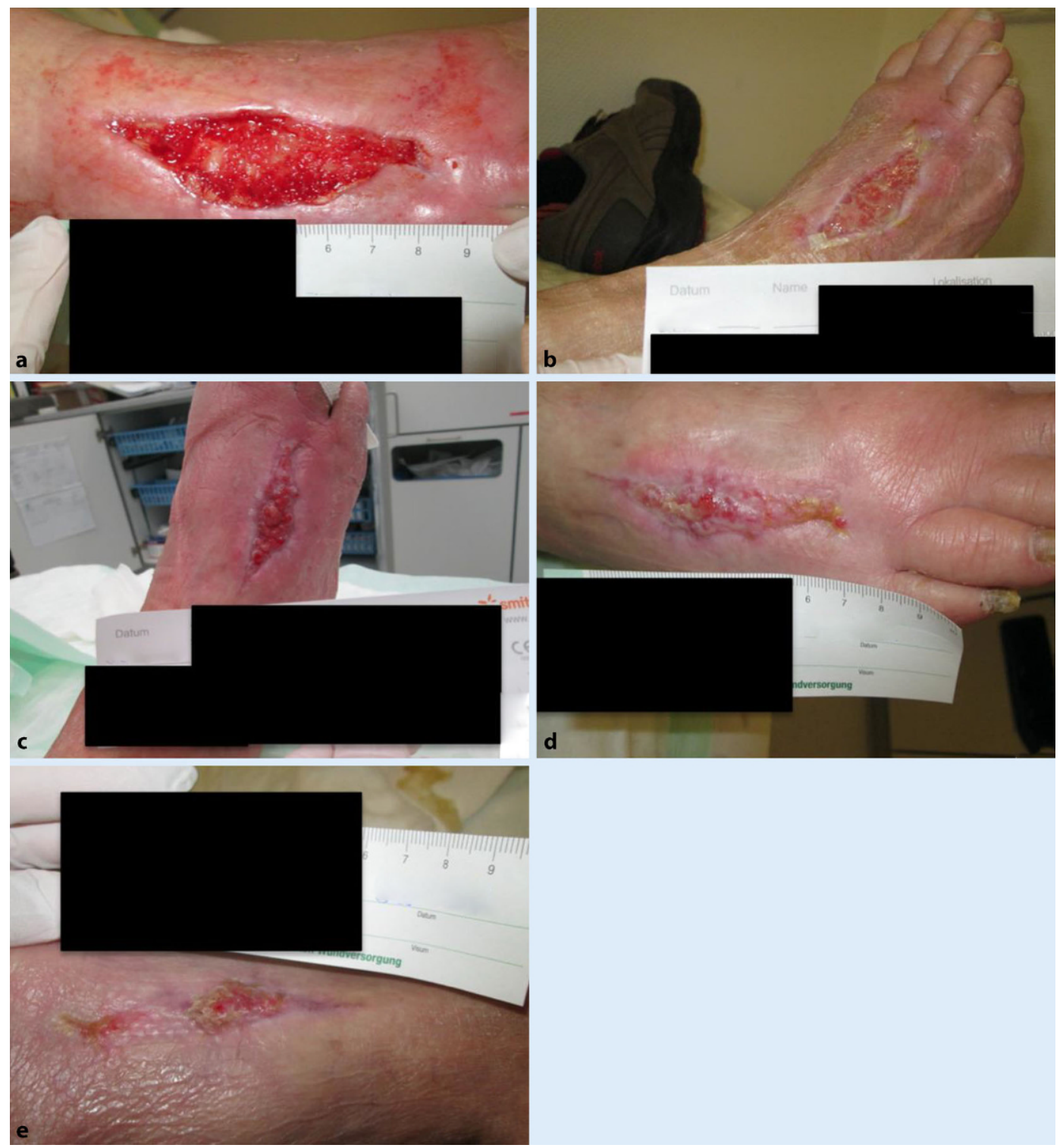

Abb. $2 \triangleleft$ Zeitlicher Ver-

lauf einer Wundbehandlung mit Nanogen Aktiv bei einer komplexen Wunde mit einem positiven Wundabstrich mit koagulasenegativen Staphylokokken nach einer Abszessbildung aufgrund einer Verletzung. Nach initialem Wunddébridement (a) zeigt sich im Verlauf (b) bereits nach ca. 6 Wochen der Behandlung Granulationsgewebe (c). Eine Epithelisierung der Wunde konnte in 9 Wochen erreicht werden (d,

e)

phase befand (- Tab. 2). Die Zeit bis zum Erreichen einer Epithelisierung betrug zwischen 4 und 40 Wochen.

Bei den zwölf Patienten, die keinen Therapieerfolg zeigten, musste die Behandlung aus verschiedenen Gründen abgebrochen werden. Diese werden im Folgenden beschrieben:

Ein Patient verstarb im Studienzeitraum an einer nicht wundassoziierten Erkrankung. Bei einem Patienten musste eine Nachamputation durchgeführt werden, im Rahmen derer die Wunde sekundär verschlossen werden konnte; somit waren keine weiteren Verbandswechsel mehr notwendig. Zwei Patienten beendeten die Behandlung auf ihren eigenen Wunsch, da sie bei den Verbandswechseln und Débri- dements zu starke Schmerzen verspürten. Eine weitere Patientin erlitt einen arteriellen Reverschluss und wollte nach erneuter Rekanalisation nicht weiter mit Nanogen Aktiv behandelt werden.

Bei vier Patienten wurde die Behandlung bei ausbleibendem Therapieerfolg beendet: Davon war bei einer Patienten die chronische Wunde durch einen Rezidiveingriff bei Z.n. Strahlentherapie bei Rektum- und Analkarzinom entstanden. Bei einer Patientin war der Wundheilungsprozess auch nach Beendigung der Lokaltherapie mit Nanogen Aktiv weiterhin ausgeblieben; in diesem Fall blieb die Wundgenese trotz weiterer Abklärung mit Ausschluss von seltenen Ulcusursachen wie Vaskulitiden oder einer Tumorerkrankung okkult. Bei zwei Patienten fand sich als einzige mögliche Ursache eine starke Besiedelung mit Pseudomonas aeruginosa; da sich jedoch bei anderen Wunden trotz dieses problematischen Keims eine Epithelisierung erreichen ließ, ist der Zusammenhang nicht geklärt.

In zwei Fällen kam es zu einer lokalen Hautreaktion mit Rötung der Umgebung, weshalb die Therapie beendet wurde.

Ein Patient stellte sich nicht wieder zum Verbandswechsel vor („lost to follow-up“).

Folgende Keime konnten in den Wunden nachgewiesen werden: Pseudomonas aeruginosa, Staphylococcus aureus, Escherichia coli, Enterococcus faecium, Enterococcus faecalis, Corynebacterium subspecies, koagulasenegative Staphy- 
Tab. 2 Studienergebnisse, aufgeteilt in Therapieerfolg und-abbruch

\begin{tabular}{|c|c|}
\hline Studienergebnisse & $n=20$ \\
\hline \multicolumn{2}{|l|}{ Therapieerfolg } \\
\hline Epithelisierung & 6 \\
\hline Granulation & 1 \\
\hline \multicolumn{2}{|l|}{ Therapieabbruch } \\
\hline Ausbleibender Therapieerfolg & 4 \\
\hline Allergische Hautreaktion & 2 \\
\hline Schmerzbedingtes Ausscheiden & 2 \\
\hline Lost to follow-up & 2 \\
\hline Sekundärnaht & 1 \\
\hline $\begin{array}{l}\text { Reverschluss nach PTA (perkutane } \\
\text { transluminale Angioplastie) }\end{array}$ & 1 \\
\hline Tod & 1 \\
\hline
\end{tabular}

Tab. 3 In den Wunden nachgewiesenes Keimspektrum; Mehrfachbesiedelung möglich

\begin{tabular}{|l|l|l|}
\hline Nachgewiesene Wundkeime & Absolute Häufigkeiten & Relative Häufigkeiten (\%) \\
\hline Staphylococcus aureus & 8 & 40 \\
\hline Pseudomonas aeruginosa & 6 & 30 \\
\hline Enterococcus faecium & 3 & 15 \\
\hline Escherichia coli & 2 & 10 \\
\hline Streptococcus dysgalactiae & 2 & 10 \\
\hline Corynebacterium species & 2 & 10 \\
\hline Klebsiella pneumoniae & 1 & 5 \\
\hline Klebsiella oxytoca & 1 & 5 \\
\hline Proteus mirabilis & 1 & 5 \\
\hline Morganella morganii & 1 & 5 \\
\hline Koagulasenegative Streptokokken & 1 & 5 \\
\hline
\end{tabular}

\begin{tabular}{|c|c|c|c|c|c|}
\hline \multicolumn{6}{|c|}{ Wundgröße } \\
\hline \multirow[t]{2}{*}{ ID } & \multirow[t]{2}{*}{$\begin{array}{l}\text { Wund- } \\
\text { lokalisation }\end{array}$} & \multirow{2}{*}{$\begin{array}{l}\begin{array}{l}\text { Wundgröße bei Therapie- } \\
\text { beginn }\end{array} \\
\mathrm{L} \times \mathrm{B} \times \mathrm{T} \text { in } \mathrm{cm}\end{array}$} & \multirow{2}{*}{$\begin{array}{l}\text { Wundgröße bei Therapie- } \\
\text { ende } \\
L \times B \times T \text { in } \mathrm{cm}\end{array}$} & \multirow{2}{*}{$\begin{array}{l}\text { Therapie } \\
\text { erfolgreich } \\
\text { beendet }\end{array}$} & \multirow{2}{*}{$\begin{array}{l}\text { Patientenzufriedenheit } \\
1 \text { (unzufrieden)-10 (sehr zufrieden) }\end{array}$} \\
\hline & & & & & \\
\hline 1 & Fuß & $4,3 \times 3,1 \times 0,7$ & $3,9 \times 3,0 \times 0,5$ & Nein & 5 \\
\hline 2 & Fuß & $6 \times 2 \times 1,3$ & $5,8 \times 1,3 \times 1$ & Nein & 1 \\
\hline 3 & Unterschenkel & $9,7 \times 3,1 \times 0,5$ & $10 \times 4 \times 0,3$ & Nein & - \\
\hline 4 & Fuß & $7 \times 2 \times 0,3$ & $2 \times 0,4 \times 0$ & $\mathrm{Ja}$ & 10 \\
\hline 5 & Unterschenkel & $11 \times 3 \times 0,5$ & $4,0 \times 0,7 \times 0$ & $\mathrm{Ja}$ & 10 \\
\hline 6 & Unterschenkel & $8,5 \times 6 \times 0,3$ & $8,5 \times 5 \times 0,3$ & Nein & 1 \\
\hline 7 & Unterschenkel & $10 \times 3,2 \times 0,8$ & $10,4 \times 3,2 \times 1$ & Nein & - \\
\hline 8 & Oberschenkel & $4,5 \times 2 \times 0,7$ & $2,8 \times 1,8 \times 0$ & $\mathrm{Ja}$ & 10 \\
\hline 9 & Unterschenkel & $11 \times 3 \times 1,5$ & $6,0 \times 3,0 \times 0$ & Nein & 6 \\
\hline 10 & Fuß & $2,8 \times 1,8 \times 0,2$ & $0,4 \times 0,2 \times 0$ & $\mathrm{Ja}$ & 10 \\
\hline 11 & Unterschenkel & $6,3 \times 5,8 \times 0,3$ & $8,5 \times 9,5 \times 0,5$ & Nein & 2 \\
\hline 12 & Fuß & $2,5 \times 2,5 \times 0,7$ & $2,5 \times 1 \times 0,2$ & $\mathrm{Ja}$ & 8 \\
\hline 13 & Unterschenkel & $13 \times 7 \times 0,3$ & $10 \times 6,5 \times 0,3$ & Nein & 1 \\
\hline 14 & Unterschenkel & $11 \times 2 \times 0,1$ & $12 \times 2 \times 0,4$ & Nein & - \\
\hline 15 & Oberschenkel & $5,0 \times 1,4 \times 0,2$ & $0,4 \times 0,3 \times 0$ & $\mathrm{Ja}$ & 8 \\
\hline 16 & Unterschenkel & $15 \times 16 \times 0,1$ & $9,3 \times 10 \times 0,1$ & Nein & 1 \\
\hline 17 & Fuß & $5,8 \times 3 \times 0,1$ & $7 \times 5,3 \times 0,1$ & Nein & 1 \\
\hline 18 & Leiste & $5,5 \times 2,2 \times 5,5$ & $5,5 \times 2,2 \times 5,5$ & Nein & 2 \\
\hline 19 & Fuß & $5,5 \times 3,5 \times 1$ & $4,5 \times 2,5 \times 0,5$ & $\mathrm{Ja}$ & 8 \\
\hline 20 & Oberschenkel & $8 \times 4 \times 1$ & $2 \times 0,5 \times 0,3$ & $\mathrm{Ja}$ & 10 \\
\hline
\end{tabular}

lokokken, Klebsiella oxytoca, Klebsiella pneumoniae, Proteus mirabilis, Streptococcus dysgalactiae, Morganella morganii (• Tab. 3). Die Abstrichentnahme zeigte in 14 Fällen eine Besiedelung mit nur einem Keim und in fünf Fällen eine Mehrfachbesiedelung. Bei einer Wunde blieben die Abstriche stets steril. Bei keiner einzigen Wunde kam es unter der zusätzlichen Verwendung von Silgen $\mathrm{Ag}$
Silberspray zu einer Veränderung des Keimspektrums oder zu einer Eradikation des Keimes.

Die Patientenzufriedenheit nach erfolgreich abgeschlossener Behandlung $(n=8)$ auf der zehnstufigen Erhebungsskala wurde fünfmal mit zehn und dreimal mit acht Punkten bewertet.
Die Veränderung der Wundgröße, der Therapieerfolg und die Patientenzufriedenheit sind in $\mathbf{0}$ Tab. 4 dargestellt.

\section{Diskussion}

Weltweit leiden circa ein bis zwei Prozent der Bevölkerung an Wunden mit gestörtem Heilungsverlauf, die sich oft sekundär auf dem Boden einer zugrunde lie- 

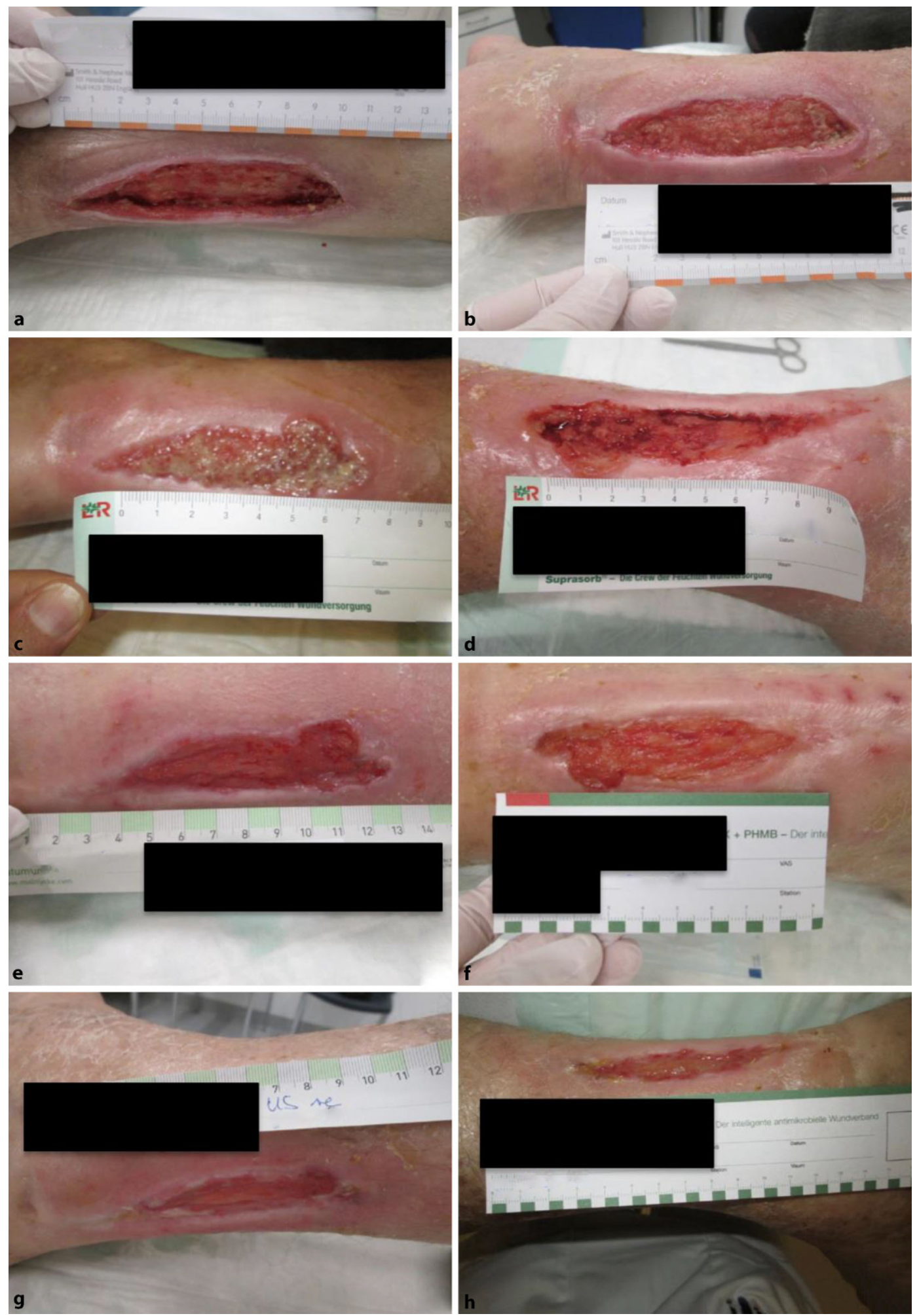

Abb. $3 \varangle$ In der Vorgeschichte wurde ein femorocruraler Bypass bei infizierter Wunde mit freiliegender Achillessehne bei pAVK-Stadium IV angelegt. Eine Keimbesiedelung mit Pseudomonas aeruginosa wurde im Wundabstrich nachgewiesen. Nach 40 Wochen konnte eine deutliche Reduktion der Wundfläche und -tiefe erreicht werden. Zeitlicher Verlauf: a Therapiebeginn, b 4 Wochen, c 8 Wochen, d 12 Wochen, e 16 Wochen, f 20 Wochen, $g 24$ Wochen, h 28 Wochen

genden Erkrankung entwickeln. Um eine effiziente Therapie zu ermöglichen, ist daher die Diagnosestellung der Grunderkrankung essenziell, um diese primär gezielt behandeln zu können. Somit ist die notwendige Voraussetzung geschaffen, um die pathologische Wundheilung normalisieren zu können. Trotzadäquater Therapie der zugrunde liegenden Pathologie zeigt jedoch ein Teil der Wunden keine Heilungstendenz, da sich in der Wunde ein Milieu gebildet hat, welches die geordnete
Ausbildung von Granulationsgewebe und Epithelisierung verhindert [10].

Zur Behandlung chronischer Wunden steht aktuell eine große Auswahl an Wundauflagen mehrerer Hersteller zur Verfügung. Trotzdem zeigte ein Cochrane-Re- 
view, in dem 42 kontrollierte randomisierte Studien analysiert wurden, keine Überlegenheit eines Verbandstyps (Hydrokolloide vs. Alginate vs. Schaumverbände vs. Hydrogele) in Bezug auf die Anzahl der geheilten Ulzerationen [7].

Im Gegensatz zu den etablierten Verbandsmaterialen stellt Nanogen Aktiv einen neuen Ansatz im Bereich der moderne Wundauflagen dar, da es den Heilungsprozess aktiv durch die Bereitstellung einer Matrix beeinflusst. Dadurch konnte in unserer Untersuchung eine Epithelisierung bzw. eine deutliche Verkleinerung von Wunden an der unteren Extremität in 7 von 20 Fällen erreicht werden.

Gerade in der Gefäßchirurgie ist der Einsatz von Matrixersatzmaterialien zur Wundbettkonditionierung vor einer MeshGraft-Deckung zur Reduktion der Wundgröße und -tiefe denkbar.

Bei den nicht erfolgreich therapierten Patienten führten verschiedene Gründe zum Therapieabbruch. Einen kausalen Zusammenhang zwischen Nanogen Aktiv und einer Verschlechterung der lokalen Wundsituation konnten wir in keinem Fall nachweisen. Da bei jedem Verbandswechsel konsequent ein chirurgisches Wunddébridement erfolgte, sind die Therapieabbrüche aufgrund der Schmerzen möglicherweise auch darauf zurückzuführen. Bei allergischer Reaktion der Wundumgebung auf die angewendeten Materialen, welche bei unserer Studie in zehn Prozent $(2 / 20)$ auftrat, wurde die Behandlung abgebrochen. Eine engmaschige klinische Kontrolle auf lokale Reaktionen sollte gerade zu Behandlungsbeginn erfolgen.

Die lokale Behandlung chronischer Wunden erfordert einen hohen Zeitaufwand, was wir auch in der vorliegenden Studie unter Anwendung von Nanogen Aktiv bestätigen konnten. Die Dauer bis zum Erreichen der Epithelisierung variierte stark und betrug im längsten Fall 40 Wochen. Bei dem erheblichen Zeitaufwand mit wöchentlicher Vorstellung zu Débridement und Verbandswechsel spielt die Compliance der Patienten eine tragende Rolle. Außerdem zeigt unsere Studie, dass die Behandlungsdauer nicht nur von der Wundgröße und -tiefe, sondern auch von der Grunderkrankung und der Keimbesiedlung beeinflusst wird. Ein positiver bakterieller Wundabstrich korreliert jedoch nicht zwangsläufig mit einer langwierigen Heilung von Beingeschwüren [9]. In unserer Beobachtungsstudie konnte eine Epithelisierung der Wunde bei einem Patient mit einer tiefen Wunde im Bereich der Achillessehne trotz Keimbesiedelung mit Pseudomonas aeruginosa erzielt werden, auch wenn die Wundheilung 40 Wochen dauerte (- Abb. 3). Allerdings kam es bei zwei Patienten, in deren Wunden ebenfalls Pseudomonas aeruginosa nachgewiesen werden konnte zu einer Verschlechterung der lokalen Verhältnisse. Ursächlich hierfür können die Virulenzproteine Pseudolysin und Protease IV des Keims sein, welche die Wundheilung signifikant behindern [8].

Über einen positiven Effekt von silberhaltigen Antiseptika auf die bakterielle KoIonisation von Wunden wurde insbesondere in der Frühphase bei Infektion berichtet [13]. Deshalb ist die adaptierte Verwendung von silberhaltigen Verbänden wichtig, um die Wundheilung zu optimieren [5]. In der vorliegenden Studie konnte jedoch keine Eradikation sowie Veränderung des Keimspektrums in den Wunden durch die Anwendung von Silgen Ag Silberspray nachgewiesen werden. Um den Effekt von Silberspray auf infizierte Wunden zu untersuchen, sind randomisierte kontrollierte Studien mit standardisierten Ergebnismaßen und längeren Nachbeobachtungszeiträumen erforderlich.

Schließlich soll sich die moderne Lokaltherapie chronischer Wunden aus der Kombination neuer Erkenntnisse der Grundlagenforschung und der klassischen Therapie ableiten. Durch ein initiales Débridement soll nekrotisches Gewebe entfernt werden; die schlecht heilende Wunde soll durch neuerliche Anfrischung aktiviert und in eine akute Wunde überführt werden. Unter diesen verbesserten Ausgangsbedingungen versucht man, einen neuen Heilungszyklus ablaufen zu lassen [10]. Dieser wichtige Aspekt kam bei drei unserer Patienten zum Tragen; da das lokale Débridement schmerzbedingt nicht toleriert werden konnte, kam es nach einer Woche zur Verschlechterung der Wunden.
Die Hauptlimitationen der vorliegenden Studie bestehen in der geringen Fallzahl und dem Fehlen einer Vergleichsgruppe. Allerdings bestand das primäre Ziel dieser Untersuchung darin, im Sinne einer Pilotserie die Effizienz und die Wirksamkeit des Präparates zu evaluieren. Weitere Untersuchungen mit dem Vergleich von Nanogen Aktiv mit anderen modernen Wundauflagen sind notwendig; dabei könnten Kriterien zur Patientenselektion erarbeitet werden, die eine gezielte Indikationsstellung für diese aufwendige Therapie ermöglichen.

\section{Fazit für die Praxis}

- Der Einsatz von Nanogen Aktiv als biologische Wundauflage kann einen positiven Effekt auf die Wundheilung bei komplexen Wunden haben.

- Nach Möglichkeit sollte primär die Behandlung der Grunderkrankung erfolgen.

- Ein initiales Débridement sollte immer noch eine Grundsäule der Wundbehandlung darstellen.

- Trotz standardisierter Anwendung des vorliegenden Verbandsmaterials sind weitere Untersuchungen notwendig, um den Einsatz im Wundmanagement optimal definieren zu können (Patientenselektion).

- Der ergänzende Nutzen von Silgen Ag Silberspray ist aktuell noch nicht abschlieBend geklärt.

- Insbesondere zu Behandlungsbeginn sollte auf allergische Hautreaktionen auf die verwendeten Materialien geachtet werden.

- Aufgrund des hohen zeitlichen Aufwandes sollte die Indikation zur Anwendung von Nanogen Aktiv individuell gestellt werden. 


\section{Korrespondenzadresse}

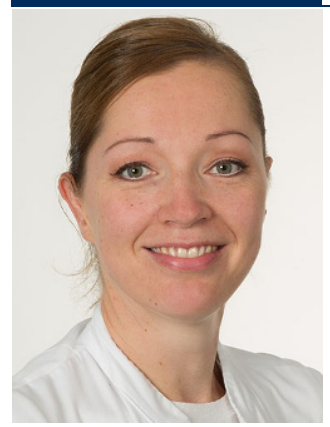

Yvonne Nicole Goßlau

Gefäßchirurgie und endovaskuläre Chirurgie, Medizinische Fakultät, Universität Augsburg Stenglinstr. 2, 86156 Augsburg, Deutschland yvonne.gosslau@uk-augsburg.de

Funding. Open Access funding enabled and organized by Projekt DEAL.

\section{Einhaltung ethischer Richtlinien}

Interessenkonflikt. R. Marnoto, T.D. Warm, K. Storl, A. Hyhlik-Dürr und Y.N. Goßlau geben an, dass kein Interessenkonflikt besteht.

Alle beschriebenen Untersuchungen am Menschen oder an menschlichem Gewebe wurden mit Zustimmung der zuständigen Ethikkommission, im Einklang mit nationalem Recht sowie gemäß der Deklaration von Helsinki von 1975 (in der aktuellen, überarbeiteten Fassung) durchgeführt. Von allen beteiligten Patienten liegt eine Einverständniserklärung vor.

Open Access. Dieser Artikel wird unter der Creative Commons Namensnennung 4.0 International Lizenz veröffentlicht, welche die Nutzung, Vervielfältigung, Bearbeitung, Verbreitung und Wiedergabe in jeglichem Medium und Format erlaubt, sofern Sie den/die ursprünglichen Autor(en) und die Quelle ordnungsgemäß nennen, einen Link zur Creative Commons Lizenz beifügen und angeben, ob Änderungen vorgenommen wurden.

Die in diesem Artikel enthaltenen Bilder und sonstiges Drittmaterial unterliegen ebenfalls der genannten Creative Commons Lizenz, sofern sich aus der Abbildungslegende nichts anderes ergibt. Sofern das betreffende Material nicht unter der genannten Creative Commons Lizenz steht und die betreffende Handlung nicht nach gesetzlichen Vorschriften erlaubt ist, ist für die oben aufgeführten Weiterverwendungen des Materials die Einwilligung des jeweiligen Rechteinhabers einzuholen.

Weitere Details zur Lizenz entnehmen Sie bitte der Lizenzinformation auf http://creativecommons.org/ licenses/by/4.0/deed.de.

\section{Literatur}

1. Deutsche Gesellschaft Für Wundheilung Und Wundbehandlunge. V.(2012)S3-Leitlinie091/001.

\section{First experiences with Bio Cellulose Nanogen Aktiv in the treatment of complex wounds}

Background: Complex wounds often pose a great challenge to both patient and therapist. In addition to the treatment of the causal disease, local therapy is essential in wound treatment. As well as the classic wound dressings, new options are now available in the form of nanomaterials.

Objective: The present study describes the influence of Nanogen Aktiv on wound healing and records patient satisfaction at the end of treatment.

Material and Methods: In the prospective single-center study, 20 patients with chronic wounds were treated with Nanogen Aktiv from February 2018 to April 2020. Demographic data, the spectrum of germs in the wounds, and clinical courses were recorded and evaluated.

Results: In 7/20 cases a reduction of the wound area could be achieved in 4-40 weeks. In the other 13 patients no therapeutic success could be recorded for various reasons. Discussion: Nanogen Aktiv can have a positive effect on the healing of complex wounds. The size and depth of the wound influence the duration of therapy. It is imperative that the underlying disease is also treated. The indication for treatment should be made on an individual basis owing to the long period of time required.

\section{Keywords}

Wound treatment $\cdot$ Extracellular matrix $\cdot$ Chronic wounds $\cdot$ PAD $\cdot$ Ulcus

Lokaltherapie chronischer Wunden bei Patienten mit den Risiken periphere arterielle Verschlusskrankheit, Diabetes mellitus, chronische venöse Insuffizienz

2. Dissemond J, Körber A, Grabbe S (2006) Differential diagnoses in leg ulcers. J Dtsch Dermatol Ges 4:627-634

3. Grimaudo MA, Concheiro A, Alvarez-Lorenzo C (2019) Nanogels for regenerative medicine. JControl Release 313:148-160

4. Heyer K, Herberger K, Protz K et al (2016) Epidemiology of chronic wounds in Germany: analysis of statutory health insurance data. Wound Repair Regen 24:434-442

5. Khansa I, Schoenbrunner AR, Kraft CT et al (2019) Silver in wound care-friend or foe?: a comprehensive review. Plast Reconstr Surg Glob Open 7:e2390

6. Kröger K, Berg C, Santosa F et al (2017) Lower limb amputation in Germany. Dtsch Arztebl Int 114:130-136

7. Palfreyman SJ, Nelson EA, Lochiel R et al (2006) Dressings for healing venous leg ulcers. Cochrane Database Syst Rev 3:CD1103

8. Prasad ASB, Shruptha P, Prabhu V et al (2020) Pseudomonas aeruginosa virulence proteins pseudolysin and protease IV impede cutaneous wound healing. Lab Invest 100:1532-1550

9. Schmidt K, Debus ES, St J et al (2000) Bacterial population of chronic crural ulcers: is there a difference between the diabetic, the venous, and the arterial ulcer? Vasa 29:62-70

10. Smola H, Eming S, Hess S et al (2001) Wundheilung und Wundheilungsstörungen: Moderne Konzepte zur Pathophysiologie und Therapie. Dtsch Arztebl 98:A 2802-2809

11. Voshege M, Wozniak G (2003) Was ist evidenzbasiert in der Behandlung chronischer Wunden? Gefässchirurgie 8:269-276

12. Winter GD (1962) Formation of the scab and the rate of epithelization of superficial wounds in the skin of the young domestic pig. Nature 193:293-294
13. Woo KY, Coutts PM, Sibbald RG (2012) A randomized controlled trial to evaluate an antimicrobial dressing with silver alginate powder for the management of chronic wounds exhibiting signs of critical colonization. Adv Skin Wound Care 25:503-508 